\title{
HAT-P-42b and HAT-P-43b
}

\section{Two inflated transiting hot Jupiters from the HATNet Survey ${ }^{\star \star \star}$}

\author{
I. Boisse ${ }^{1}$, J. D. Hartman ${ }^{2}$, G. Á. Bakos ${ }^{2, \star \star \star}$, K. Penev ${ }^{2}$, Z. Csubry ${ }^{2}$, B. Béky ${ }^{3}$, D. W. Latham ${ }^{3}$, A. Bieryla ${ }^{3}$, \\ G. Torres ${ }^{3}$, G. Kovács 4,12 , L. A. Buchhave ${ }^{5,6}$, T. Hansen ${ }^{5,6}$, M. Everett ${ }^{3}$, G. A. Esquerdo ${ }^{3}$, T. Szklenár ${ }^{3}$, E. Falco ${ }^{3}$, \\ A. Shporer ${ }^{7,8,9}$, B. J. Fulton ${ }^{10,8}$, R. W. Noyes ${ }^{3}$, R. P. Stefanik ${ }^{3}$, J. Lázár ${ }^{11}$, I. Papp ${ }^{11}$, and P. Sári ${ }^{11}$ \\ ${ }^{1}$ Centro de Astrofísica, Universidade do Porto, Rua das Estrelas, 4150-762 Porto, Portugal \\ e-mail: Isabelle.Boisse@astro.up.pt \\ 2 Princeton University, Department of Astrophysical Sciences, Princeton, NJ, USA \\ 3 Harvard-Smithsonian Center for Astrophysics, Cambridge, MA, USA \\ 4 Konkoly Observatory, Budapest, Hungary \\ 5 Niels Bohr Institute, University of Copenhagen, 2100 Copenhagen, Denmark \\ ${ }^{6}$ Centre for Star and Planet Formation, Natural History Museum of Denmark, University of Copenhagen, 1350 Copenhagen, \\ Denmark \\ 7 Division of Geological and Planetary Sciences, California Institute of Technology, Pasadena, CA 91125, USA \\ 8 LCOGT, 6740 Cortona Drive, Santa Barbara, CA, USA \\ 9 Department of Physics, Broida Hall, University of California, Santa Barbara, CA 93106, USA \\ ${ }_{10}$ Institute for Astronomy, University of Hawaii, Honolulu, HI, USA \\ 11 Hungarian Astronomical Association, Budapest, Hungary \\ 12 Department of Physics and Astrophysics, University of North Dakota, Grand Forks, ND, USA
}

Received 22 December 2012 / Accepted 14 April 2013

\section{ABSTRACT}

\begin{abstract}
Aims. We announce the discovery of two new transiting planets, and provide their accurate initial characterization.
Methods. First identified from the HATNet wide-field photometric survey, these candidate transiting planets were then followed-up with a variety of photometric observations. Determining the planetary nature of the objects and characterizing the parameters of the systems were mainly done with the SOPHIE spectrograph at the $1.93 \mathrm{~m}$ telescope at OHP and the TRES spectrograph at the $1.5 \mathrm{~m}$ telescope at FLWO.

Results. HAT-P-42b and HAT-P-43b are typical hot Jupiters on circular orbits around early-G/late-F main sequence host stars, with periods of $4.641878 \pm 0.000032$ and $3.332687 \pm 0.000015$ days, masses of $1.044 \pm 0.083$ and $0.662 \pm 0.060 M_{\mathrm{J}}$, and radii of $1.280 \pm 0.153$ and $1.281_{-0.033}^{+0.062} R_{\mathrm{J}}$, respectively. These discoveries increase the sample of planets with measured mean densities, which are needed to constrain theories of planetary interiors and atmospheres. Moreover, their hosts are relatively bright $(V<13.5)$, which facilitates further follow-up studies.
\end{abstract}

Key words. planetary systems - techniques: radial velocities - techniques: photometric - stars: individual: HAT-P-42 stars: individual: HAT-P-43

\footnotetext{
* Full Table 2 is only available in electronic form at the CDS via anonymous ftp to cdsarc.u-strasbg. fr (130.79.128.5) or via http://cdsarc.u-strasbg.fr/viz-bin/qcat?]/A+A/558/A86 $\star \star$ The photometric/spectroscopic data presented in this paper are based in part on observations carried out by the Hungarian-made Automated Telescope Network, using telescopes operated at the Fred Lawrence Whipple Observatory (FLWO) of the Smithsonian Astrophysical Observatory (SAO), and at the Submillimeter Array (SMA) of SAO, by the Tillinghast Reflector $1.5 \mathrm{~m}$ telescope and the $1.2 \mathrm{~m}$ telescope, both operated by SAO at FLWO, by the SOPHIE spectrograph mounted on the $1.93 \mathrm{~m}$ telescope at Observatoire de Haute Provence, France (runs DDT-Dec. 2011), by the Nordic Optical Telescope, operated on the island of La Palma jointly by Denmark, Finland, Iceland, Norway, and Sweden, in the Spanish Observatorio del Roque de los Muchachos of the Instituto de Astrofisica de Canarias, and by the facilities of the Las Cumbres Observatory Global Telescope. $\star \star \star$ Alfred P. Sloan Fellow and Packard Fellow.
}

\section{Introduction}

Currently, more than 800 confirmed exoplanets are known, including about 200 well-characterized transiting planets (i.e. with planetary masses and radii measured to better than $10 \%$ accuracy). Transiting extrasolar planets are crucial for exploring the physical properties of exoplanets, such as their mass-radius relationships, atmospheric composition or orbital obliquity. They provide an important first step toward a valuable comparison of extrasolar planets. These objects will be the targets of upcoming space missions designed for atmospheric characterization.

Radial velocity (RV) surveys with well defined sample limits estimated an occurrence rate of hot Jupiters in the Solar neighborhood to be around one percent (Udry \& Santos 2007; Wright et al. 2012). With a transit probability around $\sim 10 \%$, a huge number of stars have to be monitored to detect such rare objects. This is why the majority of the transiting planets were discovered by photometric surveys targeting tens of 

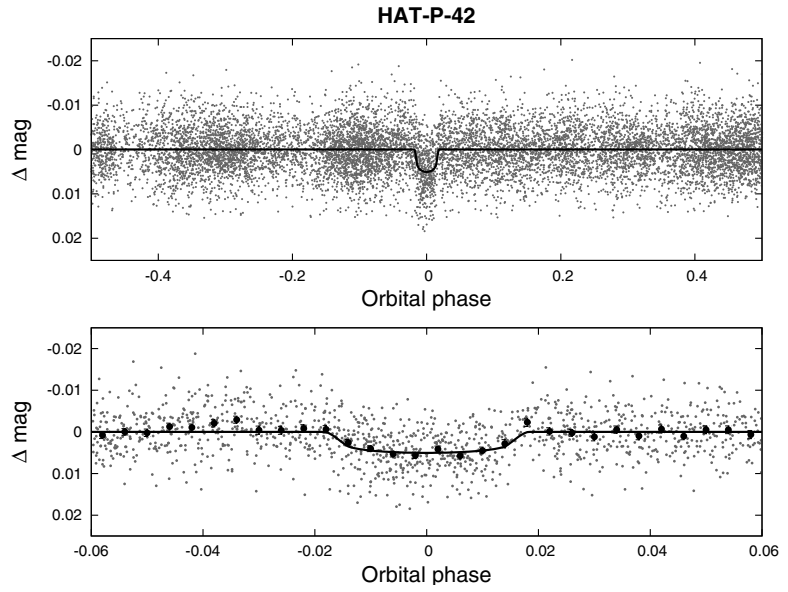
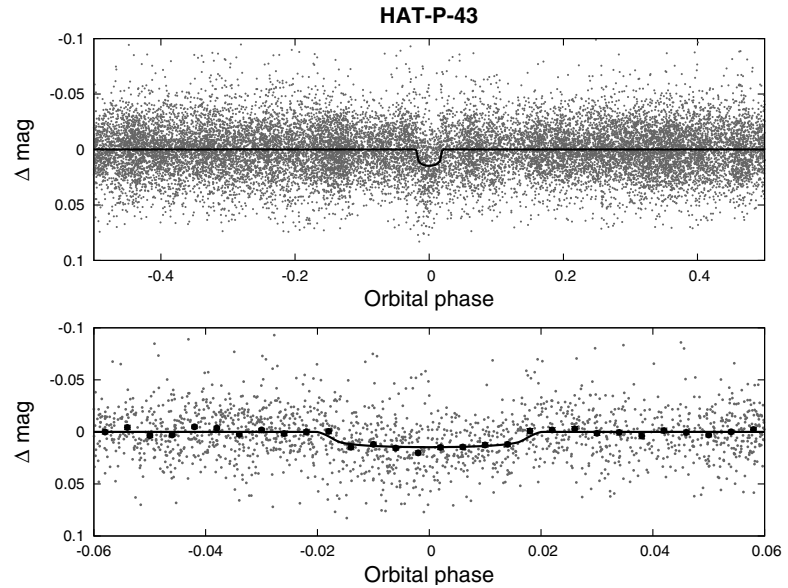

Fig. 1. HATNet light curves of HAT-P-42 (left) and HAT-P-43 (right) phase folded with the transit period. In both cases we show two panels: the top shows the unbinned light curve, while the bottom shows the region zoomed-in on the transit, with dark filled circles for the light curve binned in phase with a binsize of 0.002 . The solid line shows the model fit to the light curve.

Table 1. Summary of photometric observations.

\begin{tabular}{llccc}
\hline \hline Instrument/Field $^{a}$ & Date(s) & Number of images & Approx. cadence (min) & Filter \\
\hline HAT-P-42 & & & & \\
HAT-6/G366 & 2010 Nov.-2011 Apr. & 5325 & 3.7 & Sloan $r$ \\
HAT-9/G366 & 2010 Nov.-2011 Apr. & 4859 & 3.7 & Sloan $r$ \\
Keplercam & 2011 Nov. 16 & 176 & 1.2 & Sloan $i$ \\
Keplercam & 2012 Jan. 6 & 310 & 1.0 & Sloan $i$ \\
Keplercam & 2012 Feb. 3 & 93 & 1.2 & Sloan $i$ \\
Keplercam & 2012 Mar. 2 & 278 & 1.1 & Sloan $i$ \\
HAT-P-43 & & & & \\
HAT-5/G317 & 2010 Nov.-2011 Apr. & 4174 & 3.7 & Sloan $r$ \\
HAT-8/G317 & 2010 Nov.-2011 Apr. & 4300 & 3.7 & Sloan $r$ \\
HAT-7/G365 & 2010 Nov.-2011 May & 8262 & 3.7 & Sloan $r$ \\
HAT-8/G365 & 2011 Apr.-2011 May & 464 & 3.7 & Sloan $r$ \\
Keplercam & 2011 Nov. 17 & 68 & 3.2 & Sloan $i$ \\
Keplercam & 2012 Jan. 13 & 87 & 2.2 & Sloan $i$ \\
FTS & 2012-Feb.-16 & 226 & 1.0 & Sloan $i$ \\
Keplercam & 2012 Mar. 3 & 141 & 2.2 & Sloan $i$ \\
BOS & 2012 Mar. 3 & 109 & 2.4 & Sloan $r$ \\
Keplercam & 2012 Mar. 23 & 125 & 2.2 & Sloan $i$ \\
\hline
\end{tabular}

Notes. ${ }^{(a)}$ HAT-5, $-6,-7$, and -10 are located at FLWO in AZ, USA. HAT-8 and -9 are located at Mauna Kea Observatory in HI, USA.

thousands of stars per night. Ground-based surveys are biased toward the detection of short period and relatively large planets, i.e. hot Jupiters (with some exceptions, e.g. HAT-P-11, Bakos et al. 2010). Complementarily, space-based surveys (i.e. CoRoT and Kepler) have excelled in the detection of longer periods and smaller photometric transit depths, and hence smaller planet sizes. But, because the target stars are often relatively faint, radial-velocity follow-up to measure the planetary mass and confirm the planetary nature of the object is often not feasible for the space-based discoveries.

We present here two new transiting hot Jupiters, first identified from the Hungarian-made Automated Telescope Network wide-field photometric ground-based survey (HATNet; Bakos et al. 2004). Since 2006, HATNet has announced and published 41 transiting exoplanets, i.e. $\sim 20 \%$ of the wellcharacterized sample. These two new typical hot Jupiters, HAT-P-42b and HAT-P-43b, were partially confirmed thanks to spectroscopic observations made with SOPHIE mounted at the $1.93 \mathrm{~m}$ telescope at the Observatoire de Haute-Provence (OHP) in France and TRES at the $1.5 \mathrm{~m}$ telescope at FLWO.
The layout of the paper is as follows. Section 2 presents the diverse photometric and spectroscopic observations that lead to the detection and characterization of these systems. In the third section, we derive the stellar parameters and the planetary orbits. The characteristics of these two hot Jupiters are discussed in the last section.

\section{Observations}

\subsection{Photometric detection}

HAT-P-42 and HAT-P-43 were identified as candidate transiting planets based on photometric observations conducted by the HATNet survey (Bakos et al. 2004). These observations are summarized, together with the follow-up photometric observations, in Table 1. The data were processed and searched for transits following the procedure of Bakos et al. (2010), see also Kovács et al. (2002, 2005). Figure 1 shows the folded HATNet light curves for both systems. We give cross-identifications, and catalog photometry on an absolute scale for each system in Table 5 . 

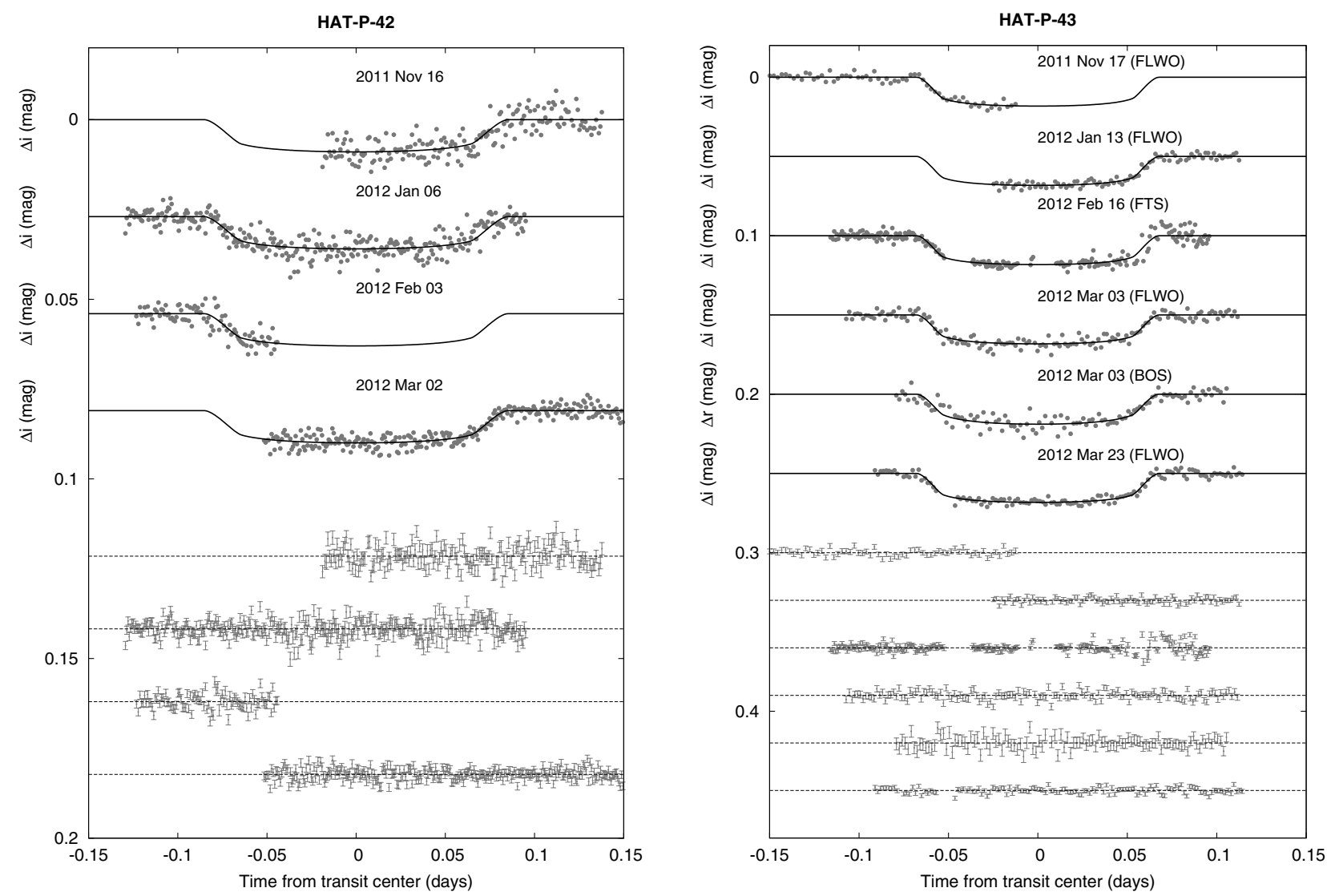

Fig. 2. Follow-up light curves for HAT-P-42 (left) and HAT-P-43 (right). For HAT-P-42 all observations were obtained with Keplercam on the FLWO $1.2 \mathrm{~m}$ telescope. For HAT-P-43 observations were obtained with Keplercam (indicated by FLWO in the figure), the Spectral CCD on the FTS $2.0 \mathrm{~m}$ (FTS), and CCD imager on the BOS $0.8 \mathrm{~m}$ (BOS). The Keplercam light curves have been corrected for trends during the modeling. The BOS and FTS light curves have also been corrected for trends, though for these light curves we only apply the external parameter decorrelation (EPD) procedure, and not the Trend Filtering Algorithm (TFA; see Bakos et al. 2010, for details). The dates of the events are indicated. Curves below the top one are displaced vertically for clarity. Our best fit from the global modeling is shown by the solid lines. Residuals from the fits are displayed at the bottom, in the same order as the top curves. The error bars represent the photon and background shot noise, plus the readout noise. They are only plotted on the residuals for readability.

\subsection{Follow-up}

We conducted follow-up spectroscopic and photometric observations of both systems to confirm their planetary natures and determine their physical properties. See Latham et al. (2009) for a more detailed description of our follow-up procedure. Below we provide specific details relevant to the discoveries of HAT-P-42b and HAT-P-43b.

\subsubsection{Reconnaissance spectroscopy}

We obtained initial high-resolution, low signal-to-noise ratio $(\mathrm{S} / \mathrm{N})$ "reconnaissance" spectra of HAT-P-42 and HAT-P-43 using the Tillinghast Reflector Echelle Spectrograph (TRES; Fúresz 2008) on the $1.5 \mathrm{~m}$ Tillinghast Reflector at the Fred Lawrence Whipple Observatory (FLWO) in AZ, USA, and using the FIbre-fed Echelle Spectrograph (FIES) on the $2.5 \mathrm{~m}$ Nordic Optical Telescope (NOT) on the island of La Palma, Spain (Djupvik \& Andersen 2010). These observations are summarized in Table 3. The data were analyzed via cross-correlation against synthetic templates as described by (Buchhave et al. 2010; Quinn et al. 2012). Both stars were found to be slowly rotating $\mathrm{G}$ dwarfs, with no evidence for composite spectra, and with low velocity variations indicating that neither object is an undiluted eclipsing binary star system.

\subsubsection{Photometry}

Photometric follow-up observations were obtained with Keplercam on the FLWO $1.2 \mathrm{~m}$, the Spectral CCD on the $2.0 \mathrm{~m}$ Faulkes Telescope South (FTS) at Siding Springs Observatory in Australia, and with the CCD imager on the $0.8 \mathrm{~m}$ Byrne Observatory at Sedgwick (BOS) telescope at Sedgwick Reserve in the Santa Ynez Valley, CA, USA. These observations are summarized in Table 1. Both FTS and BOS are operated by the Las Cumbres Observatory Global Telescope (LCOGT ${ }^{1}$; Shporer et al. 2011; Brown et al., in prep.). The Keplercam observations were reduced to light curves following the procedure of Bakos et al. (2010), while the FTS and BOS observations were reduced following the procedure of Fulton et al. (2011).

Figure 2 shows the photometric follow-up light curves for both systems together with our best-fit models after detrending as described in Sect. 3. HAT-P-42 and HAT-P-43 have photometric transit depths of 7.6 and $16.5 \mathrm{mmag}$, respectively. The data are provided in machine-readable format in Table 2.

\subsubsection{Confirmation spectroscopy}

We obtained high-resolution, high-S/N spectra of HAT-P-42 and HAT-P-43 using the SOPHIE spectrograph on the $1.93 \mathrm{~m}$

1 http://lcogt.net 
Table 2. High-precision differential photometry of HAT-P-42 and of HAT-P-43.

\begin{tabular}{lccccc}
\hline \hline Object & $\begin{array}{c}\mathrm{BJD}^{a} \\
-2455000\end{array}$ & $\mathrm{Mag}^{b}$ & $\sigma_{\mathrm{Mag}}$ & Mag $_{\text {orig }}{ }^{c}$ & Filter \\
& & & & \\
\hline HAT-P-42 & 882.87909 & 0.01318 & 0.00170 & 11.46340 & $i$ \\
& 882.88049 & 0.01025 & 0.00191 & 11.46150 & $i$ \\
& 882.88128 & 0.01064 & 0.00192 & 11.45990 & $i$ \\
& 882.88208 & 0.00410 & 0.00192 & 11.45190 & $i$ \\
& $\ldots$. & $\ldots$. & $\ldots$. & $\ldots$. & $\ldots$. \\
\hline HAT-P-43 & 883.89634 & 0.00397 & 0.00167 & 12.40490 & $i$ \\
& 883.89856 & 0.00002 & 0.00157 & 12.40130 & $i$ \\
& 883.90082 & -0.00162 & 0.00153 & 12.39990 & $i$ \\
& 883.90306 & 0.00397 & 0.00150 & 12.40600 & $i$ \\
& $\ldots$. & $\ldots$. & $\ldots$. & $\ldots$. & $\ldots$. \\
\hline
\end{tabular}

Notes. ${ }^{(a)}$ Barycentric Julian Date calculated directly from UTC, without correction for leap seconds. ${ }^{(b)}$ The out-of-transit level has been subtracted. These magnitudes have been subjected to the EPD and, in some cases, TFA procedures, carried out simultaneously with the transit fit. The TFA procedure has not been applied to the HAT-P-43 observations with $2455973<$ BJD < 2455975 or to the HAT-P-43 observations in the $r$ filter with $2455990<$ BJD $<2455991$. ${ }^{(c)}$ Raw magnitude values without application of the EPD or TFA procedures. This table is available in a machine-readable at the CDS. A portion is shown here for guidance regarding its form and content.

telescope at OHP (Bouchy et al. 2009), and of HAT-P-42 using TRES on the FLWO $1.5 \mathrm{~m}$ telescope. These observations are summarized in Table 3. The TRES observations of HAT-P-42 were simply a continuation of the first two "reconnaissance" TRES observations of this target which were already of sufficient $\mathrm{S} / \mathrm{N}$ to detect the $\sim 100 \mathrm{~m} \mathrm{~s}^{-1}$ orbital variation. Of the 13 TRES measurements, 4 with excessively low $\mathrm{S} / \mathrm{N}$ (i.e. precision $>100 \mathrm{~m} \mathrm{~s}^{-1}$ ) were excluded from the orbital analysis. While we have previously used SOPHIE to confirm HATNet planets (e.g. Bakos et al. 2007; Shporer et al. 2009), there have been significant changes to the instrument and reduction procedure. These significantly increased the RV accuracy (Perruchot et al. 2011; Bouchy et al. 2012), thus we provide a description of our observing and reduction procedures below.

We observed HAT-P-42 and HAT-P-43 with the High Efficiency fibers, yielding a resolving power of $R=\lambda / \Delta \lambda \approx$ 39000 (at $550 \mathrm{~nm}$ ). The spectra were correlated with a G2 numerical mask in order to calculate the cross-correlation function (CCF). This CCF is fitted by a Gaussian and its center gives the RV measurement (Baranne et al. 1996; Pepe et al. 2002). The bisector span values were calculated as described in Queloz et al. (2001). The error bars were derived from the CCF, following the procedure in Boisse et al. (2010), yielding a mean precision of $\sim 6 \mathrm{~m} \mathrm{~s}^{-1}$ for HAT-P-42 and $\sim 10 \mathrm{~m} \mathrm{~s}^{-1}$ for HAT-P-43. Since most of our spectra were polluted by moonlight, we corrected them following the method presented in Hébrard et al. (2008), and increased the error bars as determined empirically, leading to a mean precision of $\sim 40 \mathrm{~m} \mathrm{~s}^{-1}$ for HAT-P-42 and $\sim 30 \mathrm{~m} \mathrm{~s}^{-1}$ for HAT-P-43. A thorium-argon calibration was performed every two hours to monitor the instrumental drift mainly due to small variation of temperature and pressure in the spectrograph tank. SOPHIE is environmentally stabilized and this intrinsic drift is less than $2 \mathrm{~m} \mathrm{~s}^{-1}$ per hour. Each measurement was also corrected for this drift.

We computed the CCF using masks corresponding to different spectral types (F0, G2, K5, M5) and found no significant variation in the RV semi-amplitude. If a planet induces a RV shift, the amplitude of the variation should remain constant regardless of the spectral lines used to measure the RV. In contrast, for the case of a blend consisting of a bright target and a faint binary having different spectral types, the relative contributions to the CCF from the different components will change when a different mask is used (Santos et al. 2002; Collier Cameron et al. 2007). This result thus favors the planetary hypothesis.

Figure 3 shows the high-precision RV curves for both systems, together with our best-fit circular-orbit models. Figure 4 shows the bisector spans as a function of the RV from the SOPHIE spectra. The BIS show no significant variability or trends relative to their error bars. The BIS values are not correlated to the RV variations in agreement with the planetary hypothesis. The data are provided in Table 4.

\section{Analysis}

To rule out the possibility that either object might actually be a blended stellar eclipsing binary system, we conducted a blend analysis similar to that done in Hartman et al. (2012). We find that in both cases we cannot rule out blend scenarios based on the photometric observations alone, however all scenarios which fit the photometric data predict significant RV and BIS variations (greater than $300 \mathrm{~m} \mathrm{~s}^{-1}$ for HAT-P-42 and greater than $1 \mathrm{~km} \mathrm{~s}^{-1}$ for HAT-P-43). Such variations of the RV and BIS are ruled out by our spectroscopic observations (Fig. 4). We conclude that both objects are transiting planet systems.

Having confirmed the planetary nature of both systems, we proceeded with their analysis following the methods described in Bakos et al. (2010), with some modifications as described in Hartman et al. (2012). Briefly, this consists of: (1) inferring the stellar atmospheric parameters from the available highresolution spectra (we used the TRES spectra of HAT-P-42, and the TRES and FIES spectra of HAT-P-43, together with the stellar parameter classification (SPC) method of Buchhave et al. 2012); (2) conducting a global Markov-chain Monte Carlo (MCMC)-based modeling of the available photometric light curves and RVs (we fix the limb darkening coefficients using the tables in Claret 2004); (3) using the spectroscopically inferred stellar effective temperatures and metallicities, together with the stellar densities determined from the light curve modeling, and the Yonsei-Yale theoretical stellar evolution models (Yi et al. 2001), to determine the stellar masses, radii and ages, as well as the planetary parameters (e.g. mass and radius) which depend on these values (Fig. 5); (4) re-analyzing the high-resolution spectra fixing the stellar surface gravities to the values found in (3), and then re-iterating steps (2) and (3).

In fitting the photometric follow-up light curves, we apply the external parameter decorrelation (EPD) and trend-filtering algorithms (TFA) to correct for possible systematic errors in the photometry. As of Bakos et al. (2010) this has been done as a matter of course in analyzing all HATNet discoveries. This amounts to modeling the observed magnitude $m_{i j}$, which is the $j$ th point in light curve $i$, as:

$m_{i j}=\bar{m}_{i}+\sum_{k=1}^{N_{\mathrm{EPD}}} u_{i k} \mathrm{EPD}_{i j k}+\sum_{k=1}^{N_{\mathrm{TFA}}} v_{k} \mathrm{TFA}_{j k}+M A\left(t_{i j}, \theta\right)$

where $\bar{m}_{i}$ is the out-of-transit magnitude of light curve $i, M A$ is the Mandel \& Agol (2002) transit model, and sums including the $\mathrm{EPD}_{i j k}$ and $\mathrm{TFA}_{j k}$ terms are the EPD and TFA filters, respectively. The $M A$ model takes as input the time of 
Table 3. Summary of spectroscopic observations.

\begin{tabular}{clcccc}
\hline \hline \multicolumn{1}{c}{ Instrument } & \multicolumn{1}{c}{ Date(s) } & $\begin{array}{c}\text { Number of } \\
\text { observations }\end{array}$ & $\begin{array}{c}\text { Resolution } \\
\lambda / \Delta \lambda\end{array}$ & $\begin{array}{c}\text { Wavelength } \\
\text { coverage [A] }]\end{array}$ & $\begin{array}{l}\text { Velocity }^{a} \\
\text { precision }\end{array}$ \\
\hline HAT-P-42 & & & & & \\
FLWO 1.5 m/TRES & 2011 Oct.-2012 Jan. & 13 & 44000 & $3900-8900$ & $\sim(30+35)^{b} \mathrm{~m} \mathrm{~s}^{-1}$ \\
OHP 1.93 m/SOPHIE & 2011 Dec. 7-13 & 5 & 39000 & $3900-6900$ & $\sim(6+35)^{c} \mathrm{~m} \mathrm{~s}^{-1}$ \\
HAT-P-43 & & 2 & 46000 & $3700-7300$ & $\sim 100 \mathrm{~m} \mathrm{~s}^{-1}$ \\
NOT 2.5 m/FIES & 2011 Oct. 24-27 & 2 & 44000 & $3900-8900$ & $\sim 100 \mathrm{~m} \mathrm{~s}^{-1}$ \\
FLWO 1.5 m/TRES & 2011 Nov.-2012 Jan. & 2 & 39000 & $3900-6900$ & $\sim(10+25)^{c} \mathrm{~m} \mathrm{~s}^{-1}$ \\
OHP 1.93 m/SOPHIE & 2011 Dec. 6-13 & 6 & &
\end{tabular}

Notes. ${ }^{(a)}$ We give the median velocity precision of the observations, and not the best precision that can be attained with the given instrument. The FIES and TRES observations of HAT-P-43 were done in reconnaissance mode (lower $\mathrm{S} / \mathrm{N}$ ) which so they yield velocities that are less precise than the SOPHIE observations. ${ }^{(b)}$ A precision of $30 \mathrm{~m} \mathrm{~s}^{-1}$, plus $35 \mathrm{~m} \mathrm{~s}^{-1}$ added in quadrature to achieve a $\chi^{2}$ per degree of freedom of unity for the Keplerian fit of HAT-P-42. ${ }^{(c)}$ Values within parentheses include the sum of the theoretical noise due to the instrument $\left(6\right.$ and $10 \mathrm{~m} \mathrm{~s}^{-1}$, respectively), plus empirical errors taking into account the correction due to moonlight in the spectra.

HAT-P-42

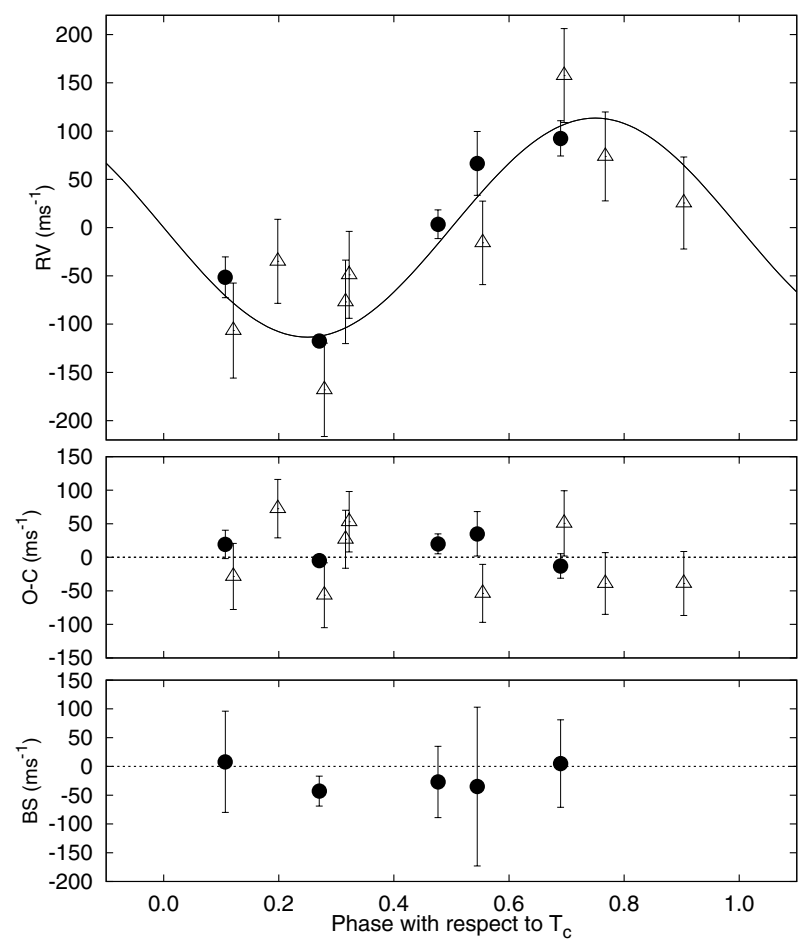

HAT-P-43

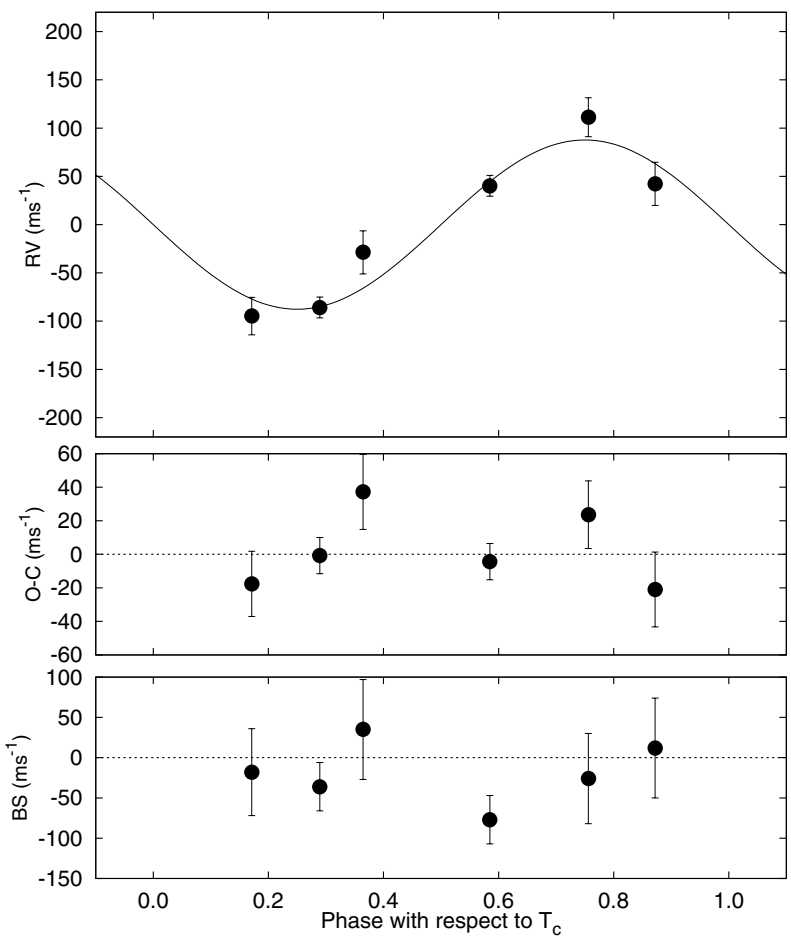

Fig. 3. High precision RV measurements and bisector spans (BIS) for HAT-P-42 (left) and HAT-P-43 (right). Filled circles show OHP $1.93 \mathrm{~m} / \mathrm{SOPHIE}$ observations, while open triangles show FLWO $1.5 \mathrm{~m} / \mathrm{TRES}$ observations. The top panels show the RV measurements as a function of orbital phase, along with our best-fit circular orbit model. Zero phase corresponds to the time of mid-transit. The center-of-mass velocity has been subtracted for each system. The middle panels show velocity $\mathrm{O}-\mathrm{C}$ residuals from the best fit circular orbit model. The bottom panels show the BIS values, with the mean values subtracted. Note the different vertical scales of the panels.

observation $t_{i j}$ and a set of physical parameters $\theta$, which includes: the radii ratio $R_{p} / R_{\star}$, the linear and quadratic limb darkening coefficients $c_{1}$ and $c_{2}$, the reciprocal of the half duration of the transit $\zeta / R_{\star}$, which is related to $a / R_{\star}$ by the expression $\zeta / R_{\star}=a / R_{\star}(2 \pi(1+e \sin \omega)) /\left(P \sqrt{1-b^{2}} \sqrt{1-e^{2}}\right)$ (Bakos et al. $2010)$, the minimum normalized impact parameter squared $b_{\min }^{2}$, two reference times of mid-transit $T_{1}$ and $T_{2}$ (we assume a strictly periodic ephemeris), and parameters characterizing the eccentricity of the orbit $\sqrt{e} \sin \omega$ and $\sqrt{e} \cos \omega$. The coefficients $u_{i k}$ and $v_{k}$ are free parameters giving the contribution from the $k$ th EPD vector for light curve $i$ and the contribution from the $k$ th TFA vector, while $\mathrm{EPD}_{i j k}$ and $\mathrm{TFA}_{j k}$ are the components of the EPD and TFA vectors, respectively. For the Keplercam follow-up light curves we use 5 EPD vectors per light curve, including the hour angle of the observation, the square of the hour angle, and three parameters describing the shape of the point spread function for that observation; for the FTS and BOS follow-up light curves we use only one, rather than three, parameters to describe the point spread function shape. We use 20 TFA vectors, corresponding to the light curves for 20 nonvariable stars observed simultaneously with each object. Due to the limited field of view, which restricts the number of potential comparison stars, we do not include the TFA terms in the models for the BOS or FTS light curves. Note that the number of free parameters in this model is much less than the number of data points in our light curves. Nonetheless by including a number of empirical detrending filters, there is a risk of overfitting the data. Because the filtering is done simultaneously with the fitting in 

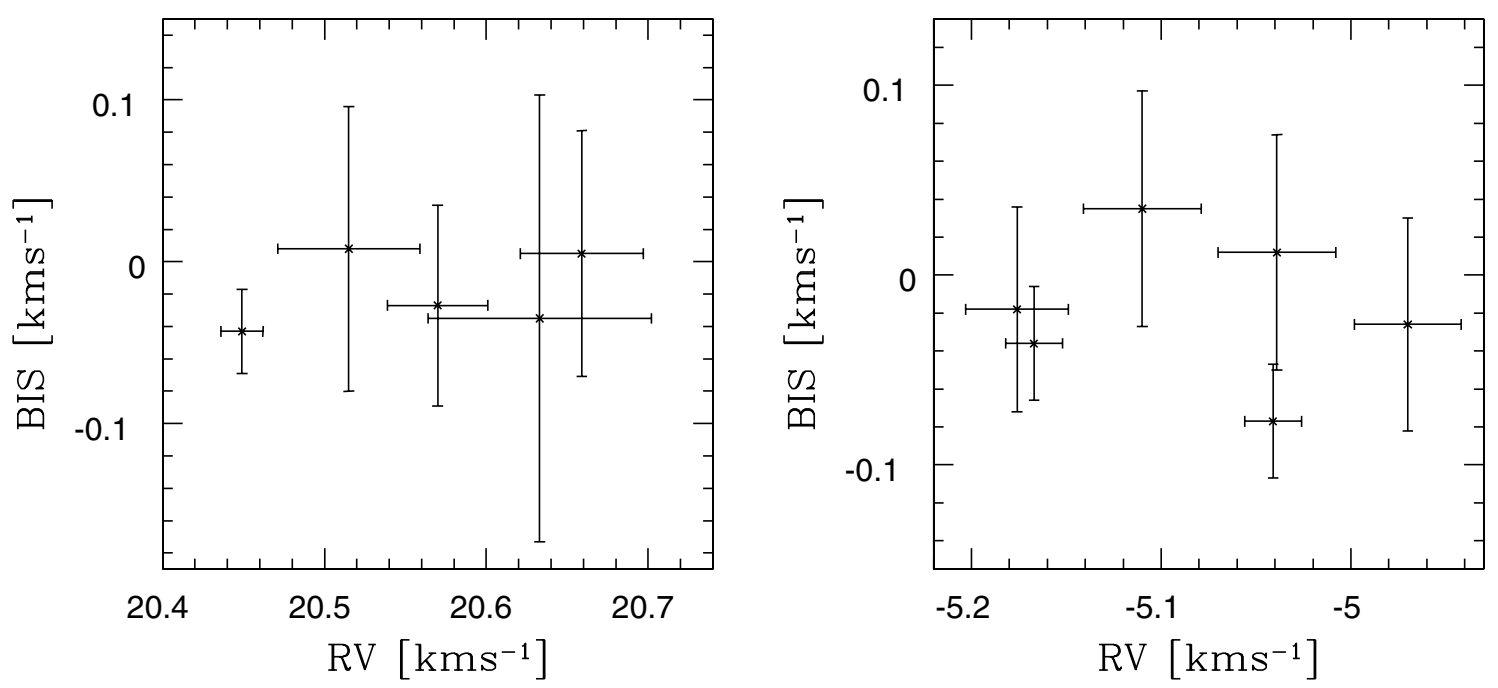

Fig. 4. Bisector spans (BIS) as a function of the high precision RV measurements for HAT-P-42 (left) and HAT-P-43 (right) from the OHP $1.93 \mathrm{~m} / \mathrm{SOPHIE}$ observations. In both panels the scale is the same in the $x$ and $y$ axes. The mean values of the BIS have been subtracted for each system.

Table 4. Relative radial velocities and bisector spans of HAT-P-42 and HAT-P-43.

\begin{tabular}{lccccc}
\hline \hline Object & $\begin{array}{c}\mathrm{BJD}^{a} \\
-2455000\end{array}$ & $\begin{array}{c}\mathrm{RV}^{b} \\
\mathrm{~m} \mathrm{~s}^{-1}\end{array}$ & $\begin{array}{c}\sigma_{\mathrm{RV}^{c}} \\
\mathrm{~m} \mathrm{~s}^{-1}\end{array}$ & $\begin{array}{c}\mathrm{BIS}^{d} \\
\mathrm{~m} \mathrm{~s}^{-1}\end{array}$ & Phase \\
\hline HAT-P-42 & 889.03539 & -47.4 & 28.4 & $\cdots$ & 0.322 \\
& 900.05380 & 159.1 & 33.8 & $\cdots$ & 0.696 \\
& 901.01864 & 27.1 & 32.4 & $\cdots$ & 0.904 \\
& 902.02682 & -105.1 & 34.6 & $\cdots$ & 0.121 \\
& 902.71983 & -112.1 & 13.0 & -43.0 & 0.270 \\
& 902.93220 & -75.4 & 25.4 & $\cdots$ & 0.316 \\
& 903.67805 & 8.9 & 31.0 & -27.0 & 0.477 \\
& 904.03850 & -14.2 & 25.4 & $\cdots$ & 0.554 \\
& 904.66696 & 97.9 & 38.0 & 5.0 & 0.690 \\
& 905.02718 & 75.3 & 29.9 & $\cdots$ & 0.767 \\
& 906.60475 & -46.1 & 44.0 & 8.0 & 0.107 \\
& 907.02711 & -33.4 & 25.9 & $\cdots$ & 0.198 \\
& 908.63608 & 71.9 & 69.0 & -35.0 & 0.545 \\
& 939.89586 & -166.5 & 33.4 & $\cdots$ & 0.279 \\
\hline HAT-P-43 & 901.68768 & -85.8 & 15.0 & -36.0 & 0.289 \\
& 902.67245 & 40.3 & 15.0 & -77.0 & 0.585 \\
& 903.62875 & 42.3 & 31.0 & 12.0 & 0.872 \\
& 904.62601 & -94.8 & 27.0 & -18.0 & 0.171 \\
& 906.57509 & 111.3 & 28.0 & -26.0 & 0.756 \\
& 908.60322 & -28.8 & 31.0 & 35.0 & 0.364 \\
\hline
\end{tabular}

Notes. Observations without a BIS value were obtained with the TRES instrument, observations with a BIS value were obtained with SOPHIE. (a) Barycentric Julian Date calculated directly from UTC, without correction for leap seconds. ${ }^{(b)}$ A zero-level $\gamma$ velocity, fitted independently for each instrument, has been subtracted from these measurements. For reference, for HAT-P-42, the SOPHIE $\gamma$ velocity from the fit was $20.561 \pm 0.014 \mathrm{~km} \mathrm{~s}^{-1}$, which is an estimate of the true recession velocity of the center of mass of the system. For TRES the value is $0.014 \pm 0.015 \mathrm{~km} \mathrm{~s}^{-1}$. As an artifact of the reduction procedure the TRES RVs were measured relative to an arbitrary zero-point, the fitted $\gamma$ velocity in this case has no physical meaning. For HAT-P-43, the SOPHIE $\gamma$ velocity from the fit was $-5.082 \pm 0.009 \mathrm{~km} \mathrm{~s}^{-1}$. $^{(c)}$ Internal errors excluding any component of astrophysical jitter. ${ }^{(d)}$ We use the relation $\sigma_{\mathrm{BIS}}=2 \sigma_{\mathrm{RV}}$ to estimate the BIS uncertainties.

our MCMC, overfitting may inflate the resulting parameter uncertainties, by allowing bad models to fit the data, but will not prevent the correct model from fitting the data, and so should not bias the results away from the true values.
In conducting the analysis we inflated the TRES RV errors for HAT-P-42 by adding in quadrature a value of $35 \mathrm{~m} \mathrm{~s}^{-1}$ to the internal errors. This "jitter" is needed to achieve a $\chi^{2}$ per degree of freedom of unity for the TRES RVs in the best-fit model. On the other hand, the SOPHIE RVs of HAT-P-42 or HAT-P-43 show in both cases a $\chi^{2}$ per degree of freedom less than one, indicating that the SOPHIE formal uncertainties may be overestimated. We completed the analysis by rescaling the error bars to obtain a $\chi^{2}$ per degree of freedom equal to one.

For both systems we conducted the analysis fixing the eccentricities to zero, as well as allowing the eccentricities to vary. For each system we find that the eccentricity is consistent with zero (the $95 \%$ confidence upper limits on the eccentricity are $e<0.2$ for HAT-P-42b, and $e<0.29$ for HAT-P-43b). Following the suggestion of Anderson et al. (2012) we adopt the parameter values associated with the fixed circular orbits. The adopted stellar parameters are given in Table 5, while the adopted planetary parameters are given in Table 7. For completeness we also provide the parameters which result when the eccentricities are allowed to be non-zero in Tables 6 and 8. Note that all the eccentric parameters are within $1 \sigma$ of the circular orbit values.

We searched for sinusoidal signals and additional transits in the light curves. Neither present a significant peak in the LombScargle periodogram (false alarm probability of $50 \%$ or more). After removing the detected in-transit data, neither light curve shows a transit signal that would pass our automated or by-eye selections.

\section{Discussion}

We have presented the discovery, confirmation and characterization of two new transiting planets. HAT-P-42b and HAT-P-43b are inflated hot Jupiters with $P=4.641878 \pm 0.000032$ and $3.332687 \pm 0.000015$ days, $M_{p}=1.044 \pm 0.083$ and $0.662 \pm$ $0.060 M_{\mathrm{J}}$, and $R_{p}=1.280 \pm 0.153$ and $1.281_{-0.033}^{+0.062} R_{\mathrm{J}}$, respectively. Assuming zero albedo and full heat redistribution, and using the stellar and planetary parameters determined by our analysis, their equilibrium temperatures are in the same regime, i.e. $T_{\text {eq }}=1428 \pm 60$ and $1361 \pm 25 \mathrm{~K}$, respectively. HAT-P-42b has larger uncertainties on its derived parameters as the transit depth is shallower due to the larger radius of the slightly 

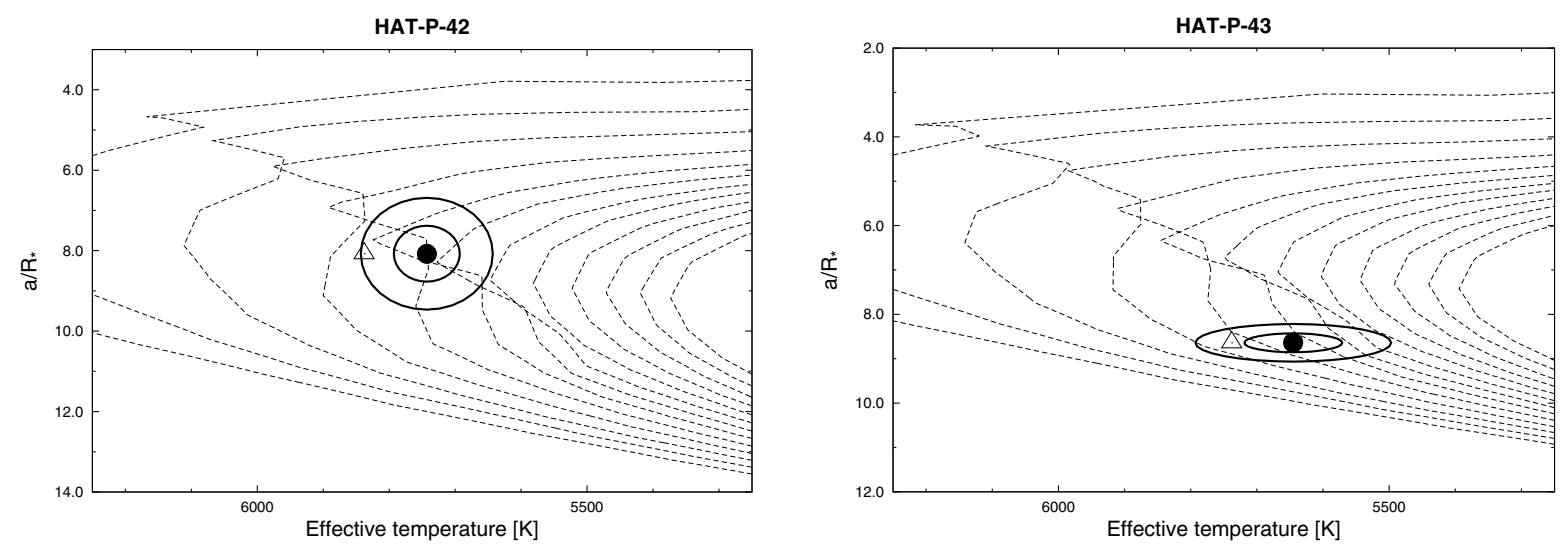

Fig. 5. Model isochrones from Yi et al. (2001) for the metallicities of HAT-P-42 (left) and HAT-P-43 (right) and ages of 1 to 13 Gyr in 1 Gyr increments (left to right). The adopted values of effective temperature, $T_{\text {eff } \star}$, and $a / R_{\star}$ are shown together with their $1 \sigma$ and $2 \sigma$ confidence ellipsoids. In each plot the initial values of $T_{\text {eff } \star}$ and $a / R_{\star}$ from the first SPC (see Sect. 3) and light curve analyses are represented with a triangle.

Table 5. Adopted stellar parameters for HAT-P-42 and HAT-P-43 assuming circular orbits.

\begin{tabular}{|c|c|c|c|}
\hline Parameter & $\begin{array}{c}\text { HAT-P-42 } \\
\text { Value }\end{array}$ & $\begin{array}{c}\text { HAT-P-43 } \\
\text { Value }\end{array}$ & Source \\
\hline \multicolumn{4}{|c|}{ Astrometric properties } \\
\hline GSC ID ....... & GSC 0232-01451 & GSC 0801-00608 & \\
\hline 2MASS-ID ... & 2MASS $09012265+0605500$ & 2MASS $08354217+1012239$ & \\
\hline RA $(J 2000) \ldots$ & $09^{\mathrm{h}} 01^{\mathrm{m}} 22.66 \mathrm{~s}$ & $08^{\mathrm{h}} 35^{\mathrm{m}} 42.18 \mathrm{~s}$ & 2MASS \\
\hline $\operatorname{Dec}(\mathrm{J} 2000) \ldots$ & $+06^{\circ} 05^{\prime} 50.0^{\prime \prime}$ & $+10^{\circ} 12^{\prime} 24.0^{\prime \prime}$ & 2MASS \\
\hline$\mu_{\mathrm{RA}}\left(\operatorname{mas~yr}^{-1}\right)$ & $-6.2 \pm 1.9$ & $-10.3 \pm 2.6$ & UCAC4 \\
\hline$\mu_{\text {Dec }}\left(\operatorname{mas~yr}^{-1}\right)$ & $-29.3 \pm 2.0$ & $-16.0 \pm 3.2$ & UCAC4 \\
\hline \multicolumn{4}{|c|}{ Spectroscopic properties } \\
\hline$T_{\mathrm{eff} \star}(\mathrm{K}) \ldots \ldots$ & $5743 \pm 50$ & $5645 \pm 74$ & $\mathrm{SPC}^{a}$ \\
\hline$[\mathrm{Fe} / \mathrm{H}] \ldots \ldots$ & $0.27 \pm 0.08$ & $0.23 \pm 0.08$ & SPC \\
\hline$v \sin i\left(\mathrm{~km} \mathrm{~s}^{-1}\right)$. & $3.5 \pm 0.5$ & $2.4 \pm 0.5$ & SPC \\
\hline$\gamma_{\mathrm{RV}}\left(\mathrm{km} \mathrm{s}^{-1}\right) \ldots$ & $20.26 \pm 0.15^{b}$ & $-4.86 \pm 0.15^{b}$ & TRES \\
\hline \multicolumn{4}{|c|}{ Photometric properties } \\
\hline$B(\mathrm{mag}) \ldots \ldots$ & $12.827 \pm 0.020$ & $14.120 \pm 0.060$ & APASS \\
\hline$V(\mathrm{mag}) \ldots . .$. & $12.168 \pm 0.030$ & $13.356 \pm 0.030$ & APASS \\
\hline$J(\mathrm{mag}) \ldots \ldots$ & $10.960 \pm 0.023$ & $12.146 \pm 0.021$ & 2MASS \\
\hline$H(\mathrm{mag}) \ldots . .$. & $10.677 \pm 0.024$ & $11.809 \pm 0.029$ & 2MASS \\
\hline$K_{\mathrm{s}}(\mathrm{mag}) \ldots \ldots$ & $10.626 \pm 0.026$ & $11.764 \pm 0.023$ & 2MASS \\
\hline \multicolumn{4}{|l|}{ Derived properties } \\
\hline$M_{\star}\left(M_{\odot}\right) \ldots$ & $1.178 \pm 0.068$ & $1.048_{-0.042}^{+0.031}$ & $\mathrm{YY}+a / R_{\star}+\mathrm{SPC}^{c}$ \\
\hline$R_{\star}\left(R_{\odot}\right) \ldots \ldots$ & $1.530 \pm 0.140$ & $1.103_{-0.021}^{+0.041}$ & $\mathrm{YY}+a / R_{\star}+\mathrm{SPC}$ \\
\hline $\log g_{\star}(\mathrm{cgs}) \ldots$ & $4.14 \pm 0.07$ & $4.37 \pm 0.02$ & $\mathrm{YY}+a / R_{\star}+\mathrm{SPC}$ \\
\hline$L_{\star}\left(L_{\odot}\right) \ldots \ldots$ & $2.27 \pm 0.43$ & $1.11 \pm 0.10$ & $\mathrm{YY}+a / R_{\star}+\mathrm{SPC}$ \\
\hline$\hat{M}_{V}(\operatorname{mag}) \ldots$ & $3.94 \pm 0.21$ & $4.73 \pm 0.11$ & $\mathrm{YY}+a / R_{\star}+\mathrm{SPC}$ \\
\hline$M_{K}(\mathrm{mag}, \mathrm{ESO})$ & $2.38 \pm 0.20$ & $3.11 \pm 0.07$ & $\mathrm{YY}+a / R_{\star}+\mathrm{SPC}$ \\
\hline Age (Gyr) .... & $5.2_{-07}^{+1.8}$ & $5.7_{-1.1}^{+1.9}$ & $\mathrm{YY}+a / R_{\star}+\mathrm{SPC}$ \\
\hline$A_{V}(\mathrm{mag})^{d} \ldots$ & $0.000 \pm 0.020$ & $0.000 \pm 0.019$ & $\mathrm{YY}+a / R_{\star}+\mathrm{SPC}$ \\
\hline Distance (pc) & $447 \pm 41$ & $542_{-16}^{+22}$ & $\mathrm{YY}+a / R_{\star}+\mathrm{SPC}$ \\
\hline
\end{tabular}

Notes. ${ }^{(a)}$ SPC $=$ "stellar parameter classification" method, described by Buchhave et al. (2012), which derives stellar atmospheric parameters from high-resolution spectra. These parameters rely primarily on SPC, but have a small dependence also on the iterative analysis incorporating the isochrone search and global modeling of the data, as described in the text. ${ }^{(b)}$ These velocities corresponds to an absolute scale references thanks to nightly observations of HD 182488 and correction of the gravitational redshift of the Sun. ${ }^{(c)} \mathrm{YY}+a / R_{\star}+\mathrm{SPC}=\mathrm{Based}$ on the YY isochrones (Yi et al. 2001), $a / R_{\star}$ as a luminosity indicator, and the SPC results. ${ }^{(d)} V$ band extinction determined by comparing the measured 2MASS and APASS photometry for the star to the expected magnitudes from the YY $+a / R_{\star}+\mathrm{SPC}$ model for the star. We use the Cardelli et al. (1989) extinction law.

more evolved host. Within $1 \sigma$, HAT-P-42b and HAT-P-43b have the same radius and $T_{\text {eq }}$. They mainly differ by their periods (semi-major axes) and by their masses (densities). HAT-P-43b is very similar to HAT-P-4b $\left(M_{p}=0.672 M_{\mathrm{J}}, P=3.05 \mathrm{~d}\right.$, $R_{p}=1.27 R_{\mathrm{J}}$, Kovács et al. 2007) with HAT-P-4 slightly more massive, hotter and more evolved star, with similar metallicity $([\mathrm{Fe} / \mathrm{H}]=0.24)$, and to OGLE-Tr-10b $\left(M_{p}=0.63 M_{\mathrm{J}}\right.$,
$P=3.1 \mathrm{~d}, R_{p}=1.25 R_{\mathrm{J}}$, Torres et al. 2008). HAT-P-42b is similar to CoRoT-19b $\left(M_{p}=1.107 M_{\mathrm{J}}, P=3.9 \mathrm{~d}, R_{p}=1.29 R_{\mathrm{J}}\right.$, Guenther et al. 2012). HAT-P-13b is the other closest analog to HAT-P-42b and HAT-P-43b but in the intermediate mass-regime $\left(M_{p}=0.851 M_{\mathrm{J}}, P=2.9 \mathrm{~d}, R_{p}=1.28 R_{\mathrm{J}}\right.$, Winn et al. $2010 \mathrm{~b}$; Bakos et al. 2009). The stellar hosts of all the previously quoted planets are slow rotators, with late F/early G spectral type, and 
Table 6. Derived stellar parameters for HAT-P-42 and HAT-P-43 allowing nonzero eccentricty.

\begin{tabular}{|c|c|c|}
\hline Parameter & $\begin{array}{c}\text { HAT-P-42 } \\
\text { Value }\end{array}$ & $\begin{array}{c}\text { HAT-P-43 } \\
\text { Value }\end{array}$ \\
\hline$M_{\star}\left(M_{\odot}\right) \ldots \ldots$ & $1.165 \pm 0.064$ & $1.043 \pm 0.048$ \\
\hline$R_{\star}\left(R_{\odot}\right) \ldots \ldots$ & $1.411 \pm 0.173$ & $1.158_{-0.072}^{+0.136}$ \\
\hline $\log g_{\star}(\mathrm{cgs}) \ldots$ & $4.21 \pm 0.09$ & $4.33 \pm 0.07$ \\
\hline$L_{\star}\left(L_{\odot}\right) \ldots \ldots$ & $1.95_{-0.39}^{+0.60}$ & $1.20_{-0.16}^{+0.33}$ \\
\hline$M_{V}(\mathrm{mag}) \ldots$. & $4.10 \pm 0.27$ & $4.65 \pm 0.21$ \\
\hline$M_{K}(\mathrm{mag}, \mathrm{ESO})$ & $2.55 \pm 0.26$ & $3.01 \pm 0.19$ \\
\hline Age (Gyr) .... & $4.9_{-0.8}^{+1.5}$ & $6.6_{-1.5}^{+2.5}$ \\
\hline$A_{V}(\mathrm{mag})^{c} \ldots$ & $0.000 \pm 0.023$ & $0.000 \pm 0.014$ \\
\hline Distance (pc).. & $414 \pm 51$ & $566_{-37}^{+67}$ \\
\hline
\end{tabular}

Notes. Quantities and abbreviations are as in Table 5, which gives our adopted values, determined assuming circular orbits. We do not list parameters that are independent of the eccentricity.

Table 7. Adopted orbital and planetary parameters for HAT-P-42b and HAT-P-43b assuming circular orbits.

\begin{tabular}{|c|c|c|}
\hline Parameter & $\begin{array}{c}\text { HAT-P-42 } \\
\text { Value }\end{array}$ & $\begin{array}{c}\text { HAT-P-43 } \\
\text { Value }\end{array}$ \\
\hline \multicolumn{3}{|l|}{ Light curve parameters } \\
\hline$P$ (days) $\ldots \ldots \ldots \ldots$ & $4.641878 \pm 0.000032$ & $3.332687 \pm 0.000015$ \\
\hline$T_{\mathrm{c}}(\mathrm{BJD})^{a}$ & $2455952.52603 \pm 0.00075$ & $2455997.37106 \pm 0.00032$ \\
\hline$T_{14}$ (days) $^{a}$ & $0.1681 \pm 0.0039$ & $0.1354 \pm 0.0011$ \\
\hline$T_{12}=T_{34}$ (days) $^{a} \ldots \ldots$ & $0.0193 \pm 0.0037$ & $0.0149 \pm 0.0009$ \\
\hline$a / R_{\star} \ldots \ldots \ldots \ldots \ldots$ & $8.08_{-0.54}^{+0.82}$ & $8.64_{-0.28}^{+0.12}$ \\
\hline$\zeta / R_{\star}^{b} \ldots \ldots \ldots \ldots \ldots$ & $13.40 \pm 0.14$ & $16.60 \pm 0.08$ \\
\hline$R_{p} / R_{\star} \ldots \ldots \ldots \ldots$ & $0.0860 \pm 0.0033$ & $0.1193 \pm 0.0018$ \\
\hline$b^{2} \ldots \ldots \ldots \ldots \ldots \ldots$ & $0.333_{-0.141}^{+0.085}$ & $0.036_{-0.024}^{+0.061}$ \\
\hline$b \equiv a \cos i / R_{\star} \ldots \ldots$ & $0.577_{-0.162}^{+0.068}$ & $0.190_{-0.096}^{+0.024}$ \\
\hline$i(\operatorname{deg}) \ldots \ldots \ldots \ldots$ & $85.9_{-0.8}^{+1.102}$ & $88.7 \pm 0.7$ \\
\hline \multicolumn{3}{|c|}{ Limb-darkening coefficients ${ }^{c}$} \\
\hline$c_{1}, i$ (linear term) & 0.2863 & 0.3003 \\
\hline$c_{2}, i$ (quadratic term) & 0.3274 & 0.3180 \\
\hline \multicolumn{3}{|l|}{$R V$ parameters } \\
\hline$K\left(\mathrm{~m} \mathrm{~s}^{-1}\right) \ldots \ldots \ldots \ldots$ & $113.5 \pm 7.9$ & $87.7 \pm 7.7$ \\
\hline$e \ldots \ldots \ldots \ldots \ldots$ & 0 (fixed) & 0 (fixed) \\
\hline \multicolumn{3}{|l|}{ Planetary parameters } \\
\hline$M_{p}\left(M_{\mathrm{J}}\right) \ldots \ldots \ldots \ldots$ & $1.044 \pm 0.083$ & $0.662 \pm 0.060$ \\
\hline$R_{p}\left(R_{\mathrm{J}}\right) \ldots$ & $1.280 \pm 0.153$ & $1.281_{-0.033}^{+0.062}$ \\
\hline$C\left(M_{p}, R_{p}\right)^{d}$ & 0.23 & 0.09 \\
\hline$\rho_{p}\left(\mathrm{~g} \mathrm{~cm}^{-3}\right) \ldots \ldots \ldots$ & $0.61_{-0.15}^{+0.34}$ & $0.39 \pm 0.05$ \\
\hline $\log g_{p}(\mathrm{cgs}) \ldots \ldots \ldots$ & $3.20 \pm 0.10$ & $3.00 \pm 0.05$ \\
\hline$a(\mathrm{AU}) \ldots \ldots \ldots \ldots$ & $0.0575 \pm 0.0011$ & $0.0443_{-0.0006}^{+0.0004}$ \\
\hline$T_{\mathrm{eq}}(\mathrm{K})^{e} \ldots \ldots \ldots \ldots$ & $1428 \pm 60$ & $1361 \pm 25$ \\
\hline$\Theta^{f} \ldots \ldots \ldots \ldots \ldots \ldots$ & $0.079_{-0.009}^{+0.014}$ & $0.044 \pm 0.004$ \\
\hline$\langle F\rangle\left(10^{8} \mathrm{erg} \mathrm{s}^{-1} \mathrm{~cm}^{-2}\right)^{g}$ & $9.38 \pm 1.56$ & $7.74_{-0.47}^{+0.65}$ \\
\hline
\end{tabular}

Notes. ${ }^{(a)}$ Reported times are in Barycentric Julian Date calculated directly from UTC, without correction for leap seconds. $T_{\mathrm{c}}$ : reference epoch of mid transit that minimizes the correlation with the orbital period. $T_{14}$ : total transit duration, time between first to last contact; $T_{12}=T_{34}$ : ingress/egress time, time between first and second, or third and fourth contact. ${ }^{(b)}$ Reciprocal of the half duration of the transit used as a jump parameter in our MCMC analysis in place of $a / R_{\star}$. It is related to $a / R_{\star}$ by the expression $\zeta / R_{\star}=a / R_{\star}(2 \pi(1+e \sin \omega)) /\left(P \sqrt{1-b^{2}} \sqrt{1-e^{2}}\right)$ (Bakos et al. 2010). ${ }^{(c)}$ Values for a quadratic law, adopted from the tabulations by Claret (2004) according to the spectroscopic (SPC) parameters listed in Table 5. ${ }^{(d)}$ Correlation coefficient between the planetary mass $M_{p}$ and radius $R_{p} .{ }^{\left({ }^{e}\right)}$ Planet equilibrium temperature averaged over the orbit, calculated assuming a Bond albedo of zero, and that flux is re-radiated from the full planet surface. $(f)$ The Safronov number is given by $\Theta=\frac{1}{2}\left(V_{\text {esc }} / V_{\text {orb }}\right)^{2}=\left(a / R_{p}\right)\left(M_{p} / M_{\star}\right)$ (see Hansen \& Barman 2007). ${ }^{(g)}$ Incoming flux per unit surface area, averaged over the orbit.

with enhanced metallicity with respect to the Sun (except for CoRoT-19, which is solar).

HAT-P-42b and HAT-P-43b are inflated hot Jupiters compared to models of coreless giant planets. The models of Fortney et al. (2007) predict a maximal radius of $\sim 1.1 R_{\mathrm{J}}$ for both planet (see their Table 4 and Fig. 7) considering the system age, planet distance and mass (maximal in the meaning of a core free planet of pure H-He). Laughlin et al. (2011) derived a simple fitting relation from the Bodenheimer et al. (2003) models to infer the expected radius of a $\mathrm{H}-\mathrm{He}$ composition planets for different masses and insolation. From the Laughlin et al. (2011) relations, we derived a predicted radius of 1.19 and $1.17 R_{\mathrm{J}}$, respectively for 
Table 8. Orbital and planetary parameters for HAT-P-42b and HAT-P-43b allowing eccentric orbits.

\begin{tabular}{|c|c|c|}
\hline Parameter & $\begin{array}{c}\text { HAT-P-42 } \\
\text { Value }\end{array}$ & $\begin{array}{c}\text { HAT-P-43 } \\
\text { Value }\end{array}$ \\
\hline \multicolumn{3}{|l|}{ Light curve parameters } \\
\hline$a / R_{\star} \ldots \ldots \ldots \ldots$ & $8.74 \pm 0.96$ & $8.20_{-0.74}^{+0.54}$ \\
\hline$\zeta / R_{\star} \ldots \ldots \ldots$ & $13.41 \pm 0.14$ & $16.62 \pm 0.09$ \\
\hline$i(\operatorname{deg}) \ldots \ldots \ldots \ldots$ & $86.6 \pm 1.1$ & $88.5_{-1.0}^{+0.7}$ \\
\hline \multicolumn{3}{|l|}{$R V$ parameters } \\
\hline$K\left(\mathrm{~m} \mathrm{~s}^{-1}\right) \ldots$ & $108.2 \pm 8.9$ & $89.4 \pm 8.6$ \\
\hline$\sqrt{e} \cos \omega \ldots$ & $-0.143_{-0.086}^{+0.119}$ & $-0.134_{-0.132}^{+0.182}$ \\
\hline$\sqrt{e} \sin \omega \ldots \ldots \ldots$ & $-0.191_{-0.157}^{-0.238}$ & $0.164_{-0.220}^{+0.162}$ \\
\hline$e \cos \omega \ldots \ldots \ldots \ldots$ & $-0.042 \pm 0.034$ & $-0.035_{-0.066}^{+0.047}$ \\
\hline$e \sin \omega \ldots \ldots \ldots \ldots$ & $-0.050_{-0.091}^{+0.063}$ & $0.041_{-0.058}^{+0.094}$ \\
\hline$e \ldots \ldots \ldots \ldots \ldots$ & $0.084 \pm 0.063$ & $0.084 \pm 0.070$ \\
\hline$\omega(\operatorname{deg}) \ldots \ldots \ldots \ldots$ & $231 \pm 62$ & $132 \pm 74$ \\
\hline \multicolumn{3}{|c|}{ Secondary eclipse parameters } \\
\hline$T_{s}(\mathrm{BJD}) \ldots \ldots \ldots$ & $2455954.723 \pm 0.101$ & $2455995.631 \pm 0.122$ \\
\hline$T_{s, 14} \ldots \ldots \ldots \ldots \ldots$ & $0.1568 \pm 0.0177$ & $0.1465 \pm 0.0240$ \\
\hline$T_{s, 12} \ldots \ldots \ldots \ldots \ldots$ & $0.0155 \pm 0.0051$ & $0.0165 \pm 0.0038$ \\
\hline \multicolumn{3}{|l|}{ Planetary parameters } \\
\hline$M_{p}\left(M_{\mathrm{J}}\right) \ldots \ldots \ldots \ldots$ & $0.981 \pm 0.097$ & $0.672 \pm 0.068$ \\
\hline$R_{p}\left(R_{\mathrm{J}}\right) \ldots \ldots \ldots \ldots$ & $1.175_{-0.146}^{+0.193}$ & $1.348_{-0.089}^{+0.163}$ \\
\hline$C\left(M_{p}, R_{p}\right) \ldots \ldots \ldots$ & 0.48 & 0.41 \\
\hline$\rho_{p}\left(\mathrm{~g} \mathrm{~cm}^{-3}\right) \ldots$ & $0.75_{-0.22}^{+0.40}$ & $0.34 \pm 0.08$ \\
\hline $\log g_{p}(\mathrm{cgs}) \ldots \ldots \ldots$ & $3.24 \pm 0.11$ & $2.96_{-0.08}^{+0.06}$ \\
\hline$a(\mathrm{AU}) \ldots \ldots \ldots \ldots$ & $0.0573 \pm 0.0010$ & $0.0443 \pm 0.0007$ \\
\hline$T_{\mathrm{eq}}(\mathrm{K})$ & $1378 \pm 75$ & $1390_{-47}^{+79}$ \\
\hline$\Theta \ldots$ & $0.081_{-0.010}^{+0.014}$ & $0.042 \pm 0.005$ \\
\hline$\langle F\rangle\left(10^{8} \mathrm{erg} \mathrm{s}^{-1} \mathrm{~cm}^{-2}\right)$ & $8.14_{-1.47}^{+2.15}$ & $8.43_{-1.05}^{+2.27}$ \\
\hline
\end{tabular}

Notes. Quantities and definitions are as in Table 7, which gives our adopted values, determined assuming circular orbits. Here we do not list parameters that are effectively independent of the eccentricity.

HAT-P-42b and HAT-P-43b, still at the $1-\sigma$ and $3-\sigma$ level from our observations.

Enoch et al. (2012) determined an empirical relation between $R_{p}, T_{\text {eq }}$, and the semi-major axis $a$ (see their Eq. (9)). This leads to an estimated radius of $1.325 \pm 0.054 R_{\mathrm{J}}$ for HAT-P-42b, matching the observed radius within the error bars. The same relations predict a radius of $1.180_{-0.023}^{+0.021} R_{\mathrm{J}}$ for HAT-P-43b, 3- $\sigma$ below our measured value. Considering also the results observed for the highly inflated hot Jupiters presented by Hartman et al. (2012), it is possible that parameters other than $T_{\text {eq }}$ and $a$ play a role in the planetary radius in the mass domain where the equation was derived for $0.5<M_{p}<2 M_{\mathrm{J}}$. Independently, Béky et al. (2011) derived a relation to determine the radius for the planets with $0.3<M_{p}<0.8 M_{\text {J }}$ that we could apply to HAT-P-43b. Their equation leads to an inferred radius of $1.133 \pm 0.044 R_{\mathrm{J}}$. Note that the predicted radius is smaller than the measured value.

Another caveat in using the above relations is the assumption of zero Bond albedo and full redistribution of the heat when calculating $T_{\text {eq }}$, whereas in reality these assumptions are unlikely to be true for all of the planets.

Few measurements per orbit lead to poor constraints on a small eccentricity. We followed the recommendation of Anderson et al. (2012), and adopted circular orbits, after a significance test on the eccentricity measurement. We note that observing the secondary transit detection is a very efficient way to characterize the shape of the orbit, that would need several tens of RV values to obtain an equivalent precision (e.g. Boisse et al. 2009; Husnoo et al. 2011). Another method to constrain a small eccentricity is the measurement of the RVs of planetary lines during the transit, but that would currently need brighter hosts to be significantly detected (Snellen et al. 2010; Montalto et al. 2011).

The Rossiter-McLaughlin effect measured via spectroscopy during the transit allows the measurement of the sky-plane projected angle between the stellar spin-axis and the planetary orbital momentum vector. With the hypothesis that the projected angle is close to zero, the expected semi-amplitude of the effect is of 26 and $34 \mathrm{~m} \mathrm{~s}^{-1}$ for HAT-P-42 and HAT-P-43, respectively.

We note that the transit of HAT-P-42b is far from equatorial $\left(b=0.577_{-0.162}^{+0.068}\right)$, which facilitates the measurement of the projected angle, since there is a smaller correlation between the stellar $v \sin i$ and the projected spin-orbit angle. According to Winn et al. (2010a), planets around stars with the $T_{\text {eff } \star}<6250 \mathrm{~K}$ are preferentially aligned (except for the systems with longest timescales for obliquity damping, e.g. HD 80606). Additionally, the (old) age of the host stars as well as the (low) mass of the planets also increase the chances that both planetary systems are well-aligned (Triaud 2011; Hébrard et al. 2011). We applied Eq. (2) of Albrecht et al. (2012) where they estimated the tidal timescale to align a planet with the stellar equator from a calibration of binary studies considering that our targets have convective envelopes (CE). These results also favor alignment for both HAT-P-42 and HAT-P-43 (the calculated timescale are equal respectively to $\tau_{\mathrm{CE}}=247$ and 558 to be reported in their Fig. 24).

To conclude, hot gas giant planets are surprisingly diverse, and the physical reasons behind the diversity and the observed 
correlations are not well understood. It is likely that the physical properties of such planets depend on a large number of parameters. Hence, there is a continued need to broaden the sample of well-characterized transiting planets in order to develop a comprehensive view of exoplanet formation, structure and evolution.

Acknowledgements. The authors thank all the staff of Haute-Provence Observatory for their contribution to the success of the ELODIE and SOPHIE projects and their support at the 1.93-m telescope. I.B. acknowledges the support of the European Research Council/ European Community under the FP7 through a Starting Grant, as well from Fundaccao para a Ciência e a Tecnologia (FCT), Portugal, through SFRH/BPD/81084/2011 and the project PTDC/CTEAST/098528/2008. We acknowledge partial funding for HATNet operations by NASA grant NNX08AF23G, and support for performing follow-up observations by NSF grant NSFAST-1108686. G.K. acknowledges the support of the Hungarian Scientific Research Foundation (OTKA) through grant K-81373. The research leading to these results has received funding from the European Community's Seventh Framework Programme (FP7/2007-2013) under grant agreement number RG226604 (OPTICON). We thank the referee for her/his comments.

\section{References}

Albrecht, S., Winn, J. N., Johnson, J. A., et al. 2012, ApJ, 757, 18 Anderson, D. R., Collier Cameron, A., Gillon, M., et al. 2012, MNRAS, 422, 1988

Bakos, G., Noyes, R. W., Kovács, G., et al. 2004, PASP, 116, 266 Bakos, G. Á., Shporer, A., Pál, A., et al. 2007, ApJ, 671, L173

Bakos, G. Á., Howard, A. W., Noyes, R. W., et al. 2009, ApJ, 707, 446

Bakos, G. Á., Torres, G., Pál, A., et al. 2010, ApJ, 710, 1724

Baranne, A., Queloz, D., Mayor, M., et al. 1996, A\&AS, 119, 373

Béky, B., Bakos, G. Á., Hartman, J., et al. 2011, ApJ, 734, 109

Bodenheimer, P., Laughlin, G., \& Lin, D. N. C. 2003, ApJ, 592, 555

Boisse, I., Moutou, C., Vidal-Madjar, A., et al. 2009, A\&A, 495, 959

Boisse, I., Eggenberger, A., Santos, N. C., et al. 2010, A\&A, 523, A88

Bouchy, F., Hébrard, G., Udry, S., et al. 2009, A\&A, 505, 853

Bouchy, F., Diaz, R. F., Hébrard, G., et al. 2012, A\&A, 549, A49

Buchhave, L. A., Bakos, G. Á., Hartman, J. D., et al. 2010, ApJ, 720, 1118
Buchhave, L. A., Latham, D. W., Johansen, A., et al. 2012, Nature, 486, 375 Cardelli, J. A., Clayton, G. C., \& Mathis, J. S. 1989, ApJ, 345, 245

Claret, A. 2004, A\&A, 428, 1001

Collier Cameron, A., Bouchy, F., Hébrard, G., et al. 2007, MNRAS, 375, 951

Djupvik, A. A., \& Andersen, J. 2010, in Highlights of Spanish Astrophysics V,

eds. J. M. Diego, L. J. Goicoechea, J. I. González-Serrano, \& J. Gorgas, 211 Enoch, B., Collier Cameron, A., \& Horne, K. 2012, A\&A, 540, A99

Füresz, G. 2008, Ph.D. Thesis, Univ. of Szeged, Hungary

Fortney, J. J., Marley, M. S., \& Barnes, J. W. 2007, ApJ, 659, 1661

Fulton, B. J., Shporer, A., Winn, J. N., et al. 2011, AJ, 142, 84

Guenther, E. W., Díaz, R. F., Gazzano, J.-C., et al. 2012, A\&A, 537, A136

Hansen, B. M. S., \& Barman, T. 2007, ApJ, 671, 861

Hartman, J. D., Bakos, G. Á., Béky, B., et al. 2012, AJ, 144, 139

Hébrard, G., Bouchy, F., Pont, F., et al. 2008, A\&A, 488, 763

Hébrard, G., Ehrenreich, D., Bouchy, F., et al. 2011, A\&A, 527, L11

Husnoo, N., Pont, F., Hébrard, G., et al. 2011, MNRAS, 413, 2500

Kovács, G., Zucker, S., \& Mazeh, T. 2002, A\&A, 391, 369

Kovács, G., Bakos, G., \& Noyes, R. W. 2005, MNRAS, 356, 557

Kovács, G., Bakos, G. Á., Torres, G., et al. 2007, ApJ, 670, L41

Latham, D. W., Bakos, G. Á., Torres, G., et al. 2009, ApJ, 704, 1107

Laughlin, G., Crismani, M., \& Adams, F. C. 2011, ApJ, 729, L7

Mandel, K., \& Agol, E. 2002, ApJ, 580, L171

Montalto, M., Santos, N. C., Boisse, I., et al. 2011, A\&A, 528, L17

Pepe, F., Mayor, M., Galland, F., et al. 2002, A\&A, 388, 632

Perruchot, S., Bouchy, F., Chazelas, B., et al. 2011, in SPIE Conf. Ser., 8151

Queloz, D., Henry, G. W., Sivan, J. P., et al. 2001, A\&A, 379, 279

Quinn, S. N., Bakos, G. Á., Hartman, J., et al. 2012, ApJ, 745, 80

Santos, N. C., Mayor, M., Naef, D., et al. 2002, A\&A, 392, 215

Shporer, A., Bakos, G. Á., Bouchy, F., et al. 2009, ApJ, 690, 1393

Shporer, A., Brown, T., Lister, T., et al. 2011, in IAU Symp. 276, eds. A. Sozzetti, M. G. Lattanzi, \& A. P. Boss, 553

Snellen, I. A. G., de Kok, R. J., de Mooij, E. J. W., \& Albrecht, S. 2010, Nature, 465,1049

Torres, G., Winn, J. N., \& Holman, M. J. 2008, ApJ, 677, 1324

Triaud, A. H. M. J. 2011, A\&A, 534, L6

Udry, S., \& Santos, N. C. 2007, ARA\&A, 45, 397

Winn, J. N., Fabrycky, D., Albrecht, S., \& Johnson, J. A. 2010a, ApJ, 718, L145

Winn, J. N., Johnson, J. A., Howard, A. W., et al. 2010b, ApJ, 718, 575

Wright, J. T., Marcy, G. W., Howard, A. W., et al. 2012, ApJ, 753, 160

Yi, S., Demarque, P., Kim, Y.-C., et al. 2001, ApJS, 136, 417 